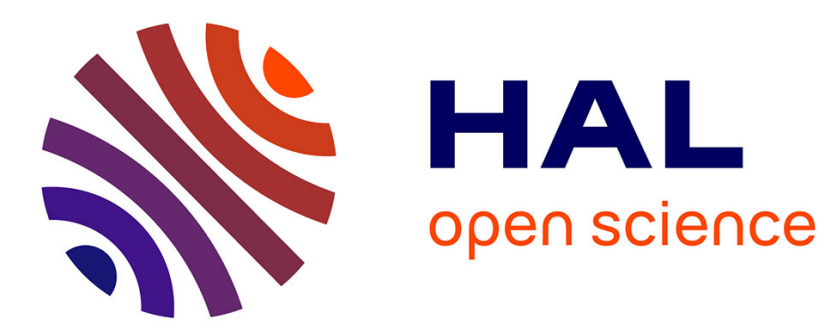

\title{
Quand les entreprises font leur marché: canaux et sélection sur les marchés du travail
}

Guillemette de Larquier, Géraldine Rieucau

\section{To cite this version:}

Guillemette de Larquier, Géraldine Rieucau. Quand les entreprises font leur marché: canaux et sélection sur les marchés du travail. Revue Française de Socio-Economie, 2014, pp.93 - 113 . hal01837185

\section{HAL Id: hal-01837185 \\ https://hal.science/hal-01837185}

Submitted on 12 Jul 2018

HAL is a multi-disciplinary open access archive for the deposit and dissemination of scientific research documents, whether they are published or not. The documents may come from teaching and research institutions in France or abroad, or from public or private research centers.
L'archive ouverte pluridisciplinaire HAL, est destinée au dépôt et à la diffusion de documents scientifiques de niveau recherche, publiés ou non, émanant des établissements d'enseignement et de recherche français ou étrangers, des laboratoires publics ou privés. 
Dernière version avant publication dans la Revue française de socio-économie $n^{\circ} 14, p$. 93-113.

\title{
Quand les entreprises font leur marché : canaux et sélection sur les marchés du travail
}

\author{
Guillemette de Larquier, \\ EconomiX (Université Paris Ouest Nanterre La Défense) \\ et Centre d'Études de l'Emploi \\ Géraldine Rieucau, \\ Centre d'Études de l'Emploi \\ et Laboratoire d'Économie Dionysien (Université Paris 8)
}

\begin{abstract}
Résumé : Pour s'adresser au marché du travail, cette «chose » a priori indéfinie, les acteurs ont besoin d'investissements de forme. Adoptant cette grille conventionnaliste, l'article caractérise le recours aux canaux de recrutement par les entreprises de quatre secteurs des services, selon deux dimensions. 1/ Ces canaux donnent-ils accès à une offre de travail sur un marché aux frontières déjà définies ou ouvrent-ils sur une offre à constituer ? 2/ Utilisentils une information au format standard ou personnalisé ? La sélection réalisée par les canaux n'est pas de même nature d'un canal à l'autre, puisqu'elle concerne des marchés du travail différemment délimités et des mains-d'œuvre différemment qualifiées.
\end{abstract}

Mots clefs : canaux de recrutement, marchés du travail, sélection, intermédiation, économie des conventions

\section{When enterprises go to the market: hiring channels and selection in labour markets}

\begin{abstract}
To address the labour market which is a priori indefinite, firms, workers and intermediaries need "investment in forms". Drawing on such a conventionalist approach, the article analyses the hiring channels used by firms in service-oriented sectors. Two questions are relevant: 1/ Do channels serve labour markets already established or do they shape the labour supply that employers are seeking? 2/ Do channels convey information in standard or personalised format? The way the channel shapes the selection depends on the ways the labour market is defined and the workforce is described.
\end{abstract}

Keywords: hiring channels, labour markets, selection, intermediation, economics of conventions 


\section{Introduction}

La littérature économique apporte des éclairages sur le rôle des canaux de recrutement (agences privées et publiques pour l'emploi, sites internet, candidatures spontanées, réseaux de relations ou encore écoles) dans la sélection de la main-d'œuvre sur le marché du travail, sans toutefois en faire un objet central. En effet, partant d'une représentation unifiée du marché du travail, les économistes standards s'intéressent plus à l'efficacité des canaux quant à leur capacité à pourvoir des emplois vacants ou des appariements de qualité [Gorter et alii, 1996 ; Cahuc, Fontaine, 2004; DeVaro, 2005; Sabatier, 2010] qu'à leur manière de sélectionner la main-d'œuvre. Les travaux sur la mesure de la discrimination à l'embauche, tels que les expérimentations via des Testing, traitent bien de la sélection réalisée sur la base des CV reçus par les entreprises, mais le canal par lequel leur parviennent les candidatures en réponse à des annonces ou de façon spontanée le plus souvent - n'est pas un objet d'études [Duguet et alii., 2007]. Cette littérature n'aborde donc pas conjointement les canaux que mobilisent les entreprises pour recruter et la façon dont la sélection est opérée. La théorie de la segmentation place quant à elle les entreprises et leurs décisions en matière d'embauche au cœur du fonctionnement du marché du travail [Dunlop 1966 ; Doeringer, Piore, 1971]. La question de la sélection des candidats est abordée au travers des caractéristiques des emplois et de la main-d'œuvre, mais celle du rôle des canaux est secondaire, à de rares exceptions près [Piore, Safford, 2007]. Dans la lignée de l'ouvrage séminal de Granovetter [1974], les travaux sociologues ont pour leur part largement démontré l'importance des réseaux sur le marché du travail, soulignant par là même que l'information sur les candidats et les emplois ne se réduit pas aux signaux du CV ou aux informations des annonces d'offre d'emploi [Ioannides, Datcher Loury, 2004]. Ils se sont également intéressés au travail des intermédiaires du placement, montrant que les cabinets de recrutement sont régis par des règles ou des « arrangements institutionnels » qui façonnent des micro-marchés centrés sur des professions, des métiers ou des secteurs [Gautié et alii. 2005] ou comment les entreprises de travail temporaire organisent la flexibilité et la disponibilité permanente de la main-d'œuvre [Chauvin, 2010]. D'après les travaux centrés sur l'intermédiaire public, la « doxa de l'activation » [Divay, 2009] et le développement des mesures de l'employabilité, ont peu à peu modelé le «tri des chômeurs » [Benarrosh, 2000] par les agents du service public de l'emploi. Cette sélection implicite repousse hors du marché du travail les demandeurs d'emploi jugés les moins employables. 
La thèse défendue dans cet article, fondée sur l'économie des conventions, soutient que les canaux mobilisés par les entreprises qui recrutent sont au cœur des mécanismes de sélection du marché du travail. Le recours à un canal particulier par l'entreprise est analysé comme le moyen pour l'entreprise de s'adresser à cette « chose informe et incertaine » qu'est le marché du travail. Dit autrement, le canal met en relation offres et demandes sur un marché, sachant qu'offres, demandes et marché du travail ne sont pas des réalités qui vont de soi pour les acteurs sans un travail préliminaire de mise en forme. En particulier, le recours à un canal suppose deux «investissements de forme $»^{1}$ [Thévenot, 1985]: une mise en forme des frontières du marché sur lequel intervient le canal et une mise en forme du langage pour qualifier les emplois et les candidats sur le marché. Ces deux investissements de forme stabilisent le recours à un canal donné et par là même le type de marché du travail que vise l'entreprise en recourant à ce canal. Il en découle que la sélection réalisée par les canaux n'est pas de même nature d'un canal à l'autre, puisqu'elle concerne des marchés du travail différemment délimités et des mains-d'œuvre différemment qualifiées. Nous développons cette thèse en prenant appui sur une enquête qualitative des pratiques de recrutement dans quatre secteurs des services (banque, conseil et services en informatique et technologies, hôtels-cafés-restaurants et grande distribution ; voir encadré méthodologique).

La première partie de cet article propose une caractérisation des canaux. Nous cherchons à comprendre leur «raison d'être » et leur diversité en nous plaçant du côté des logiques de recours des entreprises. Cela aboutit à une cartographie selon deux axes. Le premier oppose le cas où le canal est considéré par l'entreprise comme un simple « véhicule » ou «tuyau » vers un marché bien défini (par la profession ou la détention d'un diplôme par exemple) au cas où le canal est explicitement mandaté pour mobiliser, voire former, une main-d'œuvre qui n'est pas sur un marché délimité. En d'autres termes, dans le premier cas, les investissements de forme (les opérations de mise en forme) réalisés par les entreprises, l'Etat et les travailleurs

\footnotetext{
${ }^{1}$ L'investissement est défini en économie comme un détour de production qui accroît un capital (matériel, financier, humain), la nature de l'investissement et du capital étant étroitement liés. L. Thévenot [1985] propose d'élargir la notion d'investissement aux règlements, normes, contrats ou marques mis en œuvre par les entreprises et les institutions. Ces opérations de mise en forme, ces «outils», sont bien des détours de production (des «sacrifices » nous dit l'auteur) et permettent un accroissement de stabilité et de généralité dans les échanges et la production. Le code Rome (Répertoire opérationnel des métiers et des emplois) de l'agence publique pour l'emploi est un bon exemple d'investissement de forme, qui stabilise et généralise les mises en relation entre employeurs et candidats
} 
ont déjà permis de stabiliser les frontières du marché (l'offre de travail est déjà constituée). Dans le second cas, la prospection demandée au canal revient à opérer sur un marché dont les frontières ne sont pas bien définies, à ouvrir sur une offre qui n'est pas déjà là. Il s'agit davantage d'une opération «en train de se faire» de mise en forme de l'offre de maind'œuvre, où le canal joue un rôle majeur. Le second axe rend compte du langage c'est-à-dire du format de l'information adopté et privilégié par le canal pour qualifier les emplois et les candidats. On oppose notamment le format standardisé des annonces qui circulent sur internet ou qui sont mises en forme par le service public de l'emploi, au format personnalisé de l'information qui circule dans les interactions en face à face et les réseaux de relations [Thévenot, 1997]. Dans la deuxième partie de l'article, les canaux mobilisés par les entreprises des quatre secteurs étudiés sont positionnés dans le schéma dessiné par les deux axes. Selon le système d'emploi de son secteur, c'est-à-dire les règles, stabilisées ou non, de mobilisation, de mobilité et de gestion de la main-d'œuvre [Fondeur, 2013a], l'entreprise utilise un canal particulier et, ce faisant, vise l'offre de travail recherchée. Notre objectif est de montrer comment la sélection de la main-d'œuvre se fait au sein des canaux, ceux-ci prenant place sur une diversité de marchés du travail.

\section{Encadré méthodologique}

Ce travail est issu d'une recherche financée dans le cadre d'un appel à projets conjoint de la Dares et du Défenseur des droits (Halde au moment de l'appel) qui portait sur la sélectivité des pratiques de recrutement. Cette recherche s'est déroulée en 2010-2011 et a été coordonnée par le Centre d'études de l'emploi. La démarche empirique et sectorielle mettait l'accent sur les liens entre systèmes d'emploi et pratiques de recrutement [Fondeur, Larquier, 2012 ; numéro spécial de la Revue de l'Ires, 2013]. Les quatre secteurs étudiés ont été le conseil et les services en informatique et technologies, assimilé aux SSII [Fondeur, 2013b], la banque [Larquier, Tuchszirer, 2013] ; les hôtels-cafés-restaurants (HCR) [Forté, Monchatre, 2013] et la grande distribution [Rieucau, Salognon, 2013]. Les trois premiers secteurs étaient explicitement requis par les financeurs de l'étude. Nous avons demandé à ajouter le secteur de la grande distribution, par intérêt pour les pratiques de recrutement dans les secteurs à bas salaires. Malgré la crise, tous ces secteurs recrutent, sur des marchés plus ou moins en tension. Chaque monographie sectorielle comptait une dizaine d'établissements et au total, 99 entretiens semi-directifs ont été menés, auprès de recruteurs (personnels des Ressources 
Humaines, directeurs d'établissement ou employés impliqués dans le recrutement), de salariés et d'intermédiaires du marché du travail (Pôle emploi, agences d'intérim, cabinets de recrutement). Les entretiens se sont déroulés en région parisienne, à l'exception de ceux du secteur HCR, qui ont également eu lieu à Strasbourg et dans sa région. Un guide d'entretien réalisé en commun a servi de fil directeur à toutes les interviews menées. Pour chacune des quatre monographies sectorielles, une équipe de deux chercheurs menaient les entretiens. Enregistrés et transcrits à l'écrit, les entretiens ont été interprétés à partir de multiples lectures et d'allers-retours entre eux.

\section{Expliquer la diversité des canaux}

Depuis les travaux de Stigler [1962], les économistes conçoivent que la recherche sur le marché du travail est avant tout une recherche coûteuse d'information, tant pour les travailleurs que pour les employeurs, la raison d'être des agences de l'emploi étant, selon cet auteur, cette information imparfaite. Ici, nous posons que les canaux - qui regroupent les intermédiaires institutionnels, les annonces, les réseaux de relation ou encore les candidatures spontanées - ont pour raison d'être de réduire, mettre en forme, rendre maîtrisable l'incertitude du marché du travail. Selon l'économie des conventions, les mises en relation sur le marché du travail sont en effet soumises à une forte incertitude du fait de la diversité des formes d'actions et des formes d'évaluations du travail sous-jacentes [Eymard-Duvernay, Marchal, 1997 ; Bessy, Eymard-Duvernay, 1997]. Pour maîtriser cette incertitude, candidats et employeurs la mettent en forme, en mobilisant des règles, des principes ou des outils qui sont autant d'investissements de forme [Thévenot, 1985] qui les confortent dans leurs décisions et dans leurs actions. Un candidat et un employeur qui se sont rencontrés via un canal ont donc usé des mêmes règles, principes et outils. Les règles peuvent correspondre aux nomenclatures de métiers (le Rome de Pôle emploi), les principes signifier le recours systématique au même niveau de diplôme et les outils être les mots clefs ou les menus déroulants pour accéder à des offres d'emploi sur Internet. C'est bien parce qu'il s'agit de «réduire les incertitudes sans les éliminer complètement » [Bureau, Marchal, 2009, p. 586] que les conventionnalistes parlent de mise en forme de l'incertitude et non pas de solution à l'incertitude [Latsis et alii, 2010]. La grille d'analyse de cet article croise deux investissements de forme nécessaires au canal pour réduire l'incertitude : les frontières du 
marché (coproduites par les travailleurs, les entreprises, l'Etat et les canaux) et le format de l'information (qui résulte principalement du travail de mise en forme effectué par les canaux).

\subsection{Une offre de main-d'œuvre où puiser ou à constituer?}

Le premier axe de notre analyse oppose le cas où l'entreprise actionne un canal pour puiser dans une offre de travail aux contours a priori délimités, au cas où l'entreprise mobilise un intermédiaire pour constituer une offre de main-d'œuvre qui n'est pas qualifiée sur un marché particulier. En d'autres termes, il s'agit de caractériser les frontières du marché externe de l'entreprise.

Si le concept de marché interne est bien défini par Doeringer et Piore [1971], celui de marché externe l'est beaucoup moins. Dès les premières lignes de leur ouvrage, P. Doeringer et M. Piore précisent qu'il faut distinguer le marché interne du travail, régi par un ensemble de règles administratives, au marché externe du travail de la théorie économique, mais que ces deux marchés sont connectés par le biais des ports d'entrée et de sortie ${ }^{2}$. Est-ce à dire que lorsque l'entreprise recrute, elle se rend sur un marché tel qu'il est modélisé par les économistes standards ? Il y aurait un marché du travail général qui obéirait (plus ou moins) aux lois de la concurrence parfaite et, de-ci de-là, quelques îlots, des "marchés fermés » [Paradeise, 1988], qui se protègeraient de cette concurrence. Cela n'est guère satisfaisant si l'on songe qu'un côté au moins du marché externe, les postes vacants des entreprises qui recrutent, est produit par les politiques internes de ces mêmes entreprises ${ }^{3}$. Quand une entreprise recourt au marché externe, elle ne s'adresse pas à un grand marché universel mais elle mobilise un canal qui la relie à une offre de travail qu'elle juge qualifiée pour occuper un poste vacant, port d'entrée vers une carrière ascendante ou non. De fait, Bessy et Larquier [2010] ont bien montré, dans le cas français, que les intermédiaires du marché du travail sont

\footnotetext{
${ }^{2}$ «The internal market, governed by administrative rules, is to be distinguished from the external labor market of conventional economic theory where pricing, allocating, and training decisions are governed directly by economic variables. These two markets are interconnected, however, and movement between them occurs at certain jobs classifications which constitute ports of entry and exit to and from an internal labor market. » [Doeringer, Piore, 1971, chapitre 1]

${ }^{3}$ Dunlop [1966] souligne ainsi que la statistique publique quand elle cherche à mesurer les postes vacants pour analyser le fonctionnement du marché du travail ne fait qu'enregistrer le résultat des choix des entreprises : seuls les postes qui sont des ports d'entrée peuvent être publiés vacants sur le marché externe, les autres postes étant pourvus en interne.
} 
spécialisés sur des segments de main-d'œuvre. Or, selon le système d'emploi du secteur, les frontières du marché externe (les contours de l'offre de main-d'œuvre recherchée) sont soit strictement définies, soit perméables, soit encore inexistantes. Ces trois cas correspondent en fait aux trois marchés repérés par Marsden [1991] : marché professionnel, marché interne d'entreprise et marché non organisé. Les marchés professionnels sont plus vraisemblablement placés à l'est de notre premier axe (puiser dans une offre) et les marchés non organisés à l'ouest (constituer une offre), alors que les marchés internes d'entreprise peuvent être des deux côtés.

Sur les marchés professionnels, où les qualifications sont transférables d'une entreprise à l'autre quel que soit le niveau de poste, l'offre de main-d'œuvre est clairement délimitée grâce aux investissements de forme sur les qualifications ou les diplômes certifiés, négociés par les travailleurs, les entreprises et l'Etat [Paradeise, 1988]. Lors d'un recrutement, le canal ou l'intermédiaire est donc mobilisé pour atteindre un public bien ciblé. Soit ce public est nombreux et le canal va diffuser l'annonce d'emploi sur les supports lus ou visités par les professionnels recherchés; c'est le cas des informaticiens continuellement sourcés par les SSII via les job board sur Internet. Soit il s'agit d'un public plus restreint, par exemple les banquiers d'affaires expérimentés, et un chasseur de tête ira discrètement débaucher un professionnel dans une banque concurrente.

Les entreprises organisées en marchés internes qui font évoluer les qualifications grâce à la formation et aux mobilités internes, recrutent sur des ports d'entrée spécifiques et puisent dans un marché aux frontières plus perméables. Pour entrer dans un marché interne, le niveau d'études compte plus (comme marqueur d'un potentiel à évoluer) que le type précis de diplôme. Les grands groupes de nos quatre monographies ${ }^{4}$ vont régulièrement puiser dans des offres de main-d'œuvre déjà constituées (les jeunes diplômés qui ont choisi les filières qui mènent «naturellement $»^{5}$ à leur activité respective) mais ils sont prêts également à sortir de leur marché externe habituel. C'est le cas des banques de détail qui élargissent leur marché à toutes les filières courtes de l'enseignement supérieur («bac $+2 / 3 »)$, plus ou moins commerciales sans se restreindre aux formations bancaires [Larquier, Tuchszirer, 2013]. Pour

\footnotetext{
${ }^{4}$ Dans les quatre secteurs, il existe des marchés internes pour les cadres (grande distribution, hôtellerierestauration), pour les techniciens et cadres de la banque de détail et les consultants des groupes de conseils.

${ }^{5}$ Bien sûr, ce qui paraît « naturel » est le résultat d'investissements de forme passés avec le système éducatif.
} 
ce faire, elles actionnent de nombreux canaux qui ouvrent le marché, tel Pôle emploi, ou elles organisent des opérations spéciales, du type «portes ouvertes ». Dans ce cas-là, les entreprises s'ouvrent à une main-d'œuvre qui n'est pas qualifiée par la détention d'un titre ou d'un diplôme précis, mais qu'elles formeront elles-mêmes après l'embauche. La formation peut également être coproduite par l'entreprise et l'intermédiaire : les chaînes de l'hôtellerierestauration, face aux pénuries d'employés qualifiés, établissent ainsi des partenariats avec les CFA (Centre de Formation des Apprentis) pour former et constituer l'offre de main-d'œuvre recherchée.

Enfin, le dernier marché repéré par Marsden [1991], non organisé, correspond au cas où aucun investissement de forme n'a été fait pour qualifier les travailleurs, a priori parfaitement substituables. Les entreprises sont prêtes à capter toute main-d'œuvre et à la juger sur des compétences qui n'ont pas valeur de qualification pour délimiter un marché. Le canal qui permet d'atteindre une telle main-d'œuvre, doit donc mettre en forme la qualité des candidats qu'il présente à l'entreprise. Tel est le cas de la MRS (Méthode de Recrutement par Simulation) de Pôle emploi qui évalue les habilités des candidats pour être employés de libreservice, indépendamment de leur expérience. Tel est également le cas de l'employé d'un restaurant qui recommande un membre de sa famille pour occuper un emploi saisonnier. Le canal des candidatures spontanées déposées en mains propres dans un magasin est également typique des canaux qui interviennent sur un marché externe sans frontières a priori.

\subsection{Un format de l'information «standard» ou «personnalisé »?}

Le deuxième axe de notre schéma reprend l'hypothèse selon laquelle à chaque type de mise en relation via un canal est associée une façon de mettre en forme et de véhiculer l'information sur les qualités du candidat et les exigences de l'employeur. Cette hypothèse prend appui sur le travail séminal d'Albert Rees [1966], qui différencie les canaux selon le nombre de candidatures procurées et la qualité des informations délivrées. Il distingue les canaux de recherche dite «extensive» de ceux de recherche dite «intensive». Dans le premier cas, les canaux procurent un grand nombre de candidatures et une information limitée sur les candidats, à l'exemple des annonces d'offre d'emplois. Dans le cas d'une recherche intensive, ils fournissent des informations approfondies et détaillées sur un petit nombre de candidats, à l'image des réseaux de relation. Recherches extensive et intensive peuvent s'articuler lorsque le recrutement se bâtit autour d'une relation de confiance entre une agence 
pour l'emploi et les entreprises. Dans la lignée de ce travail précurseur, nous accordons du prix au «format » [Thévenot, 1985, 1997] dans lequel circule l'information sur le marché du travail. Les repères de la coordination entre les acteurs du marché $(\mathrm{CV}$, recommandations etc.) et l'évaluation des candidats ne sont pas les mêmes selon qu'ils circulent à distance [Lagarenne, Marchal, 1995] et dans un format standardisé ou selon qu'ils sont mis en récit ou dans un écrit non standard et plus empirique et transporté de proche en proche [EymardDuvernay, Marchal, 1997 ; Marchal, Rieucau, 2010]. Le choix du format de l'information dépend pour beaucoup des moyens en ressources RH disponibles pour la suite de l'évaluation (personnel, outils, etc.) [Larquier, Marchal, 2012].

Dotées de ressources et de services RH importants, les banques ont de ce point de vue une facilité à actionner des canaux qui fournissent un grand nombre de candidatures, arrivant sous formes de résumés écrits, typiquement des $\mathrm{CV}$ et des dossiers, qui transmettent directement l'information du diplôme. Les recruteurs de la banque peuvent ensuite ajouter une information plus détaillée sur la qualité des candidats, car leur processus de sélection, basé sur des tests et des entretiens, ne fait que commencer à ce stade. Pour les SSII, les CVthèques des jobboards véhiculent l'information sur les diplômes et les outils informatiques maîtrisés dans un format standard. En outre, le langage adopté sur ces sites, que ce soit pour consulter les CVthèques ou publier une annonce, est familier aux informaticiens, aux candidats et aux recruteurs, de même que leur mode d'utilisation : poster un $\mathrm{CV}$, rafraîchir régulièrement sa page, créer des alertes, etc. A contrario, l'embauche des employés dans la grande distribution et la restauration se fait plutôt via des canaux qui atténuent le poids du CV et privilégient un format personnalisé de l'information. C'est le cas lorsque les candidatures sont déposées en mains propres par les candidats, dans la mesure où une discussion informelle est souvent improvisée à ce moment-là. Le format personnalisé est également privilégié lorsqu'un salarié en poste recommande un de ses proches, pratique courante dans ces secteurs où le recrutement se fait fréquemment en urgence. L'organisation et les ressources allouées jouent ici également. Candidatures spontanées et réseaux permettent en effet aux entreprises de recruter rapidement et à peu de frais [Bessy, Marchal, 2009]. Le format personnalisé de l'information est typique d'une organisation qualifiée d'informelle, où le recrutement est pris en charge par les directeurs des établissements ou leurs adjoints, qui sont des acteurs présents au quotidien sur le terrain, connaissant bien le marché du travail local et n'étant pas des spécialistes des ressources humaines [Lockyer, Scholarios, 2004 ; Nickson et alii, 2012 ]. 


\subsection{Des mises en forme soumises à la critique des employeurs}

La délimitation du marché (déjà constitué ou non) et le format partagé de l'information sont nécessaires à l'usage stabilisé du canal par l'entreprise : l'incertitude sur les candidats est réduite, ou plutôt mise en forme, par le canal, l'entreprise n'ayant plus qu'à sélectionner parmi eux. A contrario, s'il n'y a pas accord sur les manières de constituer et de qualifier l'offre, sélectionner parmi les candidatures portées par le canal paraît risqué. L'employeur critiquera et remettra en question la pertinence du canal pour recruter. Il peut y avoir désaccord sur la qualité de la main-d'œuvre sélectionnée par le canal, soit parce qu'elle semble trop éloignée du marché dans lequel l'entreprise souhaite puiser, soit parce que le langage utilisé par le canal pour qualifier cette main-d'œuvre ne semble pas pertinent à l'entreprise.

Pôle emploi est l'archétype du canal qui « ouvre » sur un marché du travail, puisque l'une de ses premières missions de service public est de placer les chômeurs les plus éloignés de l'emploi. Cette mission se heurte cependant aux a priori négatifs des recruteurs vis-à-vis des «profils ANPE» : les candidats qui passent par Pôle emploi seraient ceux qui peinent à trouver du travail, des cas socialement difficiles, ils feraient moins d'effort de présentation, auraient des difficultés de compréhension ou des comportements inadaptés. Même si les recruteurs sont conscients de la tâche difficile qui incombe à l'opérateur public : «Pôle emploi, par rapport à ça, n'est pas fautif non plus, ils sont obligés d'accueillir des gens et eux pareil, ils sont obligés de nous les envoyer » (employeur HCR, chaîne), leur jugement global est que le «profil ANPE » manque de motivation : «Aujourd'hui, j'ai encore des gens qui viennent de Pôle emploi déposer un CV qui me demandent à ce qu'on leur mette un tampon comme quoi ils ont déposé le CV. Ils ne cherchent pas d'emploi ces gens-là! » (Directeur de supermarché indépendant). Une telle défiance vis-à-vis de Pôle emploi n'est pas de mise s'il existe une relation pérenne avec l'agent qui a présélectionné les candidatures : «Après ... c'est une question de confiance. Quand ils voient que le discours qui est tenu se concrétise par des actes, ils ont confiance » (agent de Pôle emploi). Ainsi, si l'agent de Pôle emploi est connu et apprécié, s’il appartient au sens large au réseau professionnel, alors le recruteur ne remet pas en cause la pertinence des candidatures reçues par ce canal.

Cette difficulté à déplacer les frontières du marché ne concerne pas que l'opérateur public. Par exemple, ce cabinet de recrutement opérant sur un segment étroit d'un marché 
professionnel, celui des profils techniques et expérimentés du secteur bancaire, dit vouloir ouvrir à de nouveaux profils, sans toutefois y parvenir :

« Pour éviter le système du clonage, pour aérer un petit peu la réflexion de notre client, qu'il ne soit pas uniquement sur des rails, même s'il est très obtus et qu'il veut absolument le spécialiste machin qui est passé par tel endroit, qui est sorti de telle école, etc., nous, on met toujours des idées latérales, pour avoir un champ. [...] Nous allons lui apporter une longue liste avec tous les gens qui correspondent exactement à ce qu'il cherche et nous allons ajouter làdedans des idées latérales, par exemple des profils plus généralistes mais très managers. [...] Et alors à ce moment-là notre client dit «ah, je suis désolé, mais moi je veux tel type de gars qui tralala, et donc désolé, donc on raye tous ceux qui ne sont pas dans le cadre que je vous ai donné », et moi je dis «monsieur le client, vous n'avez pas voulu, ok, alors vous allez voir vos gars, vous allez voir qu'ils sont tous formatés de la même façon » [...] on peut aussi reconnaître que notre client a parfaitement raison et qu'il est arrivé à trouver son bonheur. » (Consultant Cabinet de recrutement, secteur bancaire)

Le discours de ce consultant fait écho à un phénomène analysé par Gautié et alii [2005], qui soutiennent une thèse sensiblement différente sur la stratégie du cabinet. Dans sa shortlist, ce dernier introduirait toujours un ou deux candidats «atypiques » afin de faire paraitre plus rare encore le profil exactement souhaité. Et lors de la dernière sélection, le client retient le «clone » qui semble d'autant mieux s'apparier au poste que les autres en sont éloignés. Le cabinet de recrutement semble donc mobilisé pour aller puiser dans un vivier très étroit et non pas pour prospecter vers des horizons nouveaux.

Les tensions surgissent également $d u$ fait d'une incompatibilité entre le format de l'information adopté par les entreprises et celui du canal. Une telle différence peut expliquer en partie le non recours à Pôle emploi. Les agents de Pôle emploi produisent en effet une information standardisée [Fretel, 2013], induite par la gestion rarement personnalisée des fichiers de demandeurs d'emploi et des demandes des entreprises, dont le premier interlocuteur peut être le salarié d'une plate-forme téléphonique. Très souvent, ce ne sont pas les mêmes personnes qui reçoivent les offres et les demandes d'emploi, d'où la nécessité d'un langage commun, qui, pour mettre en correspondance les attentes des uns et des autres, a de bonnes chances d'être uniformisé. Ainsi, les compétences des demandeurs d'emploi sont traduites en un certain nombre de variables prédéfinies : diplômes, années d'expérience dans des emplois (eux-mêmes traduits en un code Rome), recherche à temps plein/partiel d'emplois, là encore traduits dans des codes Rome, etc. Or, ce langage peut être très éloigné de certains employeurs qui expriment les compétences recherchées dans un registre beaucoup 
plus empirique, renvoyant souvent à des aptitudes comportementales. L'incompréhension est parfois telle que Pôle emploi est accusé de rendre les annonces incompréhensibles : «Dans leurs annonces, il y a des filtres et c'est risqué... et puis il y a des numéros que seuls eux comprennent, nous ça ne nous dit rien. Leur site est trop compliqué, c'est trop rigide, tout est nomenclaturé »(Directeur de magasin). Certains recruteurs supportent mal que Pôle emploi complète ou réécrive «leurs » offres, tandis que l'opérateur public tient à ajouter des informations sur le poste et à gommer les libellés potentiellement discriminatoires.

\section{La mise en forme de la sélection par les canaux}

Les canaux mobilisés par les entreprises des secteurs enquêtés sont positionnés sur les quatre cadrans dessinés par les deux axes introduits dans la première partie. Lorsqu'un canal est utilisé dans plusieurs secteurs (par exemple, les écoles), seules les observations les mieux renseignées lors des entretiens sont présentées. Par ailleurs, les «cas emblématiques » de chaque secteur sont exposés : certains canaux sont en effet spécifiques à un secteur (par exemple la recommandation par un salarié récompensée par une prime dans les SSII); ils ne représentent pas forcément un volume important de candidatures et de recrutements mais ne sont cités que par les acteurs d'une monographie sectorielle. Chaque cadran nous permet de décrire les caractéristiques du marché du travail, selon que les mises en relation se font via tel ou tel canal. Notre objectif est de monter comment les canaux mettent en forme la sélection sur les quatre marchés du travail ainsi mis en évidence. 


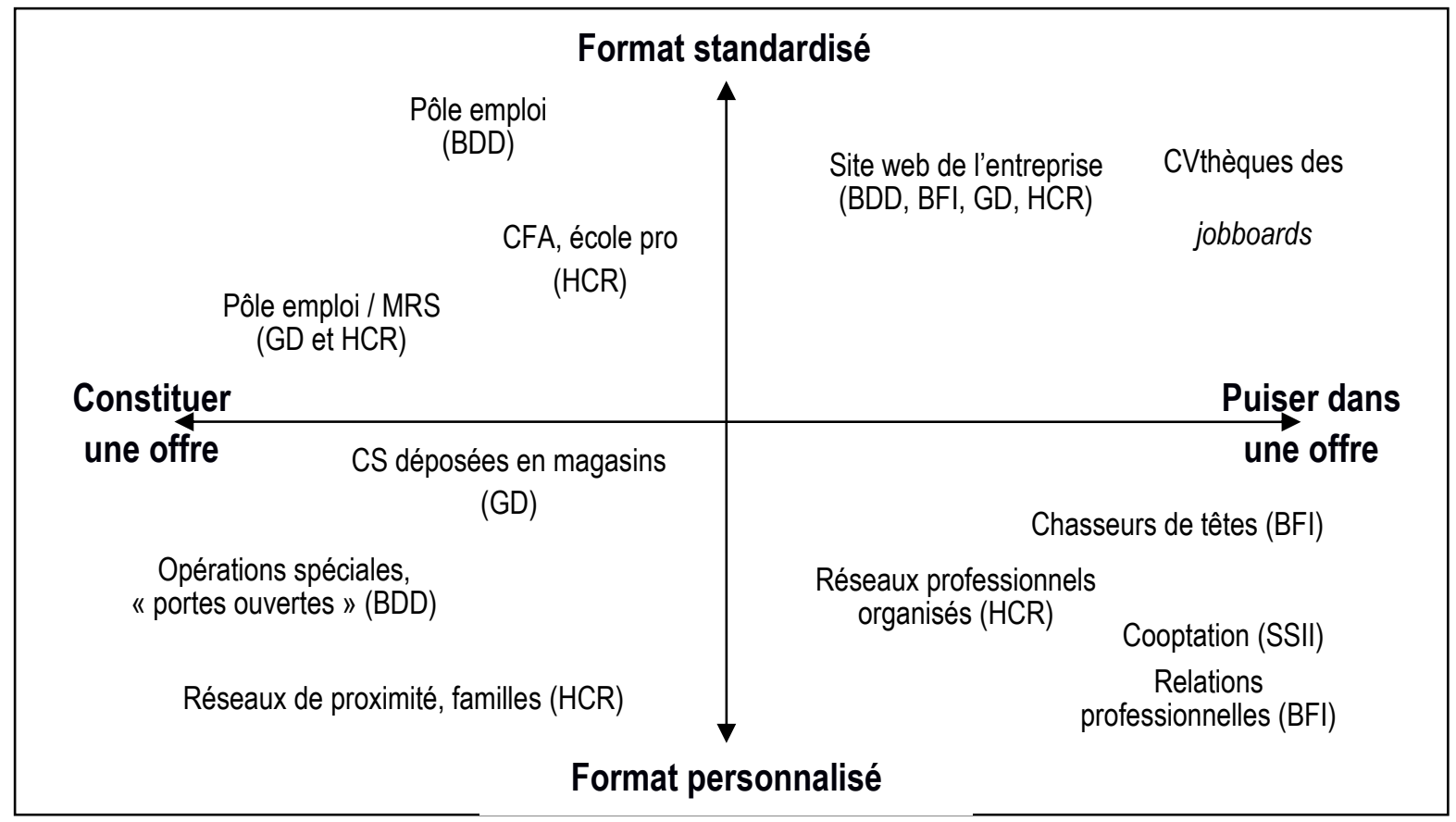

Légende $:$ BDD = banques de détail $;$ BFI = banques de financement et d'investissement $;$ SSII = sociétés de services en ingénierie informatique ; HCR = hôtels, cafés et restaurants; GD = grande distribution.

\subsection{Envoyer et recevoir des informations sur un marché du travail standard}

Dans le cadran nord-est, les entreprises reçoivent des candidatures du marché via les réponses aux annonces d'offre d'emploi, les CVthèques et les candidatures déposées sur Internet. Les groupes enquêtés ont tous une section « recrutement » sur leur site corporate sur le web, où figurent les offres d'emploi. Les candidats peuvent aussi démarcher directement le site (en créant un compte d'utilisateur) et en se positionnant sur l'un des postes mis en ligne. Lorsqu'une entreprise mobilise de tels canaux pour accéder au marché, celui-ci est conçu comme un espace stabilisé où circule une information standardisée sur des offres et des demandes, relativement génériques. Dans ce cas-là, l'entreprise attend du canal qu'il soit un «tuyau » qui fait circuler de l'information sans la déformer. Or, cela nécessite une mise en forme préliminaire de l'information pour qu'elle rentre dans ces tuyaux. La sélection opérée repose alors sur des critères stabilisés, voire durcis, tels le diplôme et l'expérience, qui peuvent entretenir l'exclusion sur le marché du travail.

Sur ce cadran, les contours de ce que l'on entend par «bon candidat » sont inscrits dans des repères qui tirent leur légitimité dans le fait d'être construits dans un monde professionnel ou scolaire. Pour postuler, il faut déjà parler la langue du monde auquel on veut appartenir et 
connaître ses codes : maîtriser l'anglais, reconnaître les intitulés des métiers de la banque, décrypter les langages et les outils du monde de l'informatique sur les jobboards des SSII, avoir le diplôme ou détenir l'expérience pour appartenir au «métier» dans la grande distribution et l'hôtellerie-restauration. Il s'agit bien de puiser dans une offre déjà constituée, aux frontières bien définies par les qualifications, celles des diplômés et des profils expérimentés du secteur, repères présents dans les annonces et les CV qui sont d'autant plus figés qu'ils circulent sur Internet. Sur ce media en effet, le recrutement peut être très outillé. Ainsi, lorsque les candidats à un poste dans une SSII remplissent en ligne les rubriques préconçues «les agents recruteurs, ce sont les mots clefs enregistrés» (Responsable recrutement, SSII). Par la mise en rubriques, Internet induit une augmentation des requis demandés dans les annonces, par rapport aux annonces publiées dans la presse [Marchal et alii, 2007]. L'exclusion opérée par les canaux de ce cadran peut de fait être très prononcée. Ne pas correspondre aux standards requis revient en effet à ne pas pouvoir postuler et il n'y a guère de possibilités de négocier les règles, de passer entre les mailles du filet de la toile. Internet multiplie le nombre de postulants, rendant la concurrence d'autant plus aiguë. Lorsqu'ils sont sollicités, les intermédiaires (Pôle emploi, jobboards, voire Apec) jouent un simple rôle de «diffuseur $»^{6}$ [Bessy, Larquier, 2010], ils ne remettent pas en cause les pratiques. Ils ne peuvent faire bouger les lignes et les contours des profils demandés ; comme cela peut être le cas dans le cadran situé à l'ouest.

A certains égards, ce marché du travail est proche de celui que décrit la théorie économique : les intermédiaires et plus largement les canaux, sont là pour fluidifier le marché, diffuser l'information et accélérer les rencontres [Autor, 2009]; l'information est produite sous forme de «signaux » [Spence, 1973] émis dans les CV des candidats et qui informent sur leur productivité. C'est au prisme de la discrimination potentielle - qui apparaît lorsque l'on s'écarte des signaux de la productivité tels que le diplôme ou l'expérience - que la sélection est abordée par la littérature standard [Duguet et alii, 2007]. L'argument de nondiscrimination est d'ailleurs présent dans le registre des acteurs qui se positionnent sur ce

\footnotetext{
${ }^{6}$ L'opérateur public a de multiples activités ; il peut simplement diffuser les annonces, comme démarcher les entreprises et les mettre en relation avec les chercheurs d'emploi, voire élaborer avec elles la MRS (Méthode de Recrutement par Simulation) [Larquier et alii, 2013]. Cette diversité des activités n'est pas incompatible avec la rationalisation croissante des dispositifs d'accompagnement des chercheurs d'emploi mise en évidence par Fretel [2013].
} 
cadran. C'est l'agent de Pôle emploi qui vérifie, avant de les diffuser, que les annonces ne comportent pas de critères discriminatoires. C'est pour éviter les jugements arbitraires et discriminatoires susceptibles de s'exprimer lorsque les personnes déposent leur CV que les responsables RH d'un groupe de distribution alimentaire instaurent un système de recrutement centralisé, où la sélection se fait principalement sur les CV reçus via Internet. Cependant, parce qu'elle privilégie les signaux du CV (diplôme, expérience), une sélection à distance dans ce secteur à bas salaires peut exclure les personnes non qualifiées et non expérimentées. Une telle sélection induit aussi des formes de discrimination indirecte, ne serait-ce que parce que tout le monde ne maîtrise pas l'outil Internet [Rieucau, 2013].

\subsection{Demander à un intermédiaire institutionnel de façonner une nouvelle main-d'œuvre}

Dans le cadran nord-ouest, le langage parlé par l'entreprise et le canal prend également un format standardisé car, ici, l'entreprise mandate des canaux qui sont des institutions (Pôle emploi ou des écoles), produisant une information standardisée sur les candidats et les emplois. Mais ces institutions ont pour mission de trouver des candidats qui ne sont pas sur un marché standard. Le plus souvent, l'entreprise dit avoir des difficultés de recrutement et cherche donc à mobiliser une main-d'œuvre qui ne vient pas à elle par les canaux classiques du cadran précédent. Le canal est alors un intermédiaire (ce ne sera jamais seulement un outil comme Internet) avec lequel s'élabore un partenariat pour sélectionner une main-d'œuvre, qui n'était pas a priori qualifiée, et qui doit le devenir grâce à la garantie apportée par l'intermédiaire.

Ainsi, pour ne pas se priver de bons commerciaux (qui ne pensent pas à faire carrière dans la banque et à aller sur les sites corporate), une banque de détail parisienne va chercher à sortir de son marché naturel (les jeunes diplômés des filières bancaires) en faisant appel à Pôle emploi. Le langage codifié de Pôle emploi est jugé efficace pour filtrer les candidatures de chargés d'accueil qui seront traitées en continu par les services RH de la banque. La conseillère de Pôle emploi est appréciée par les recruteurs de cette banque parce que les CV qu'elle envoie sont ajustés à ses attentes pour trouver des commerciaux en dehors des filières habituelles. Sa bibliothèque de requêtes informatiques pour rechercher des candidats dans les fichiers régionaux et nationaux est le résultat d'une vingtaine d'années de mise en relations pour les différentes banques franciliennes. Elle reprend même les CV et lettres de motivation des candidats pour les rapprocher des attentes des recruteurs, elle change par exemple un titre 
général de $\mathrm{CV}^{7}$ et endosse le rôle de traductrice entre une offre de travail a priori non totalement ajustée et la demande de la banque. En cela, elle ouvre le marché à des profils qui, selon elle, ne seraient peut-être pas évalués en passant par les pages recrutement du site corporate.

De leur côté, les entreprises de la grande distribution et de l'hôtellerie-restauration sollicitent Pôle emploi, les Centres de formations d'apprentis (CFA) ou autres écoles professionnelles, pour avoir accès à une offre de travail qui n'est pas déjà là. C'est ce que permet la MRS (Méthode de Recrutement par Simulation) conçue par Pôle emploi pour placer des demandeurs d'emploi non qualifiés, envers lesquels les recruteurs ont un certain nombre de préjugés. En partenariat avec des enseignes et souvent à l'occasion de l'ouverture d'un nouvel établissement, Pôle emploi conçoit des exercices reflétant les conditions de travail et destinés à repérer les habiletés nécessaires pour occuper les postes. Par exemple, pour un poste d'employé de libre-service, il faut remplir des rayons en temps limité, dans une ambiance sonore rappelant celle du magasin, tout en étant interrompu par des clients ou des collègues fictifs. Les candidats sont évalués sur leurs habilités techniques comme relationnelles et ceux qui réussissent sont convoqués sur le lieu de travail pour un entretien, qui se déroule sans CV [Bouchardeau, Capelier, 2010 ; Larquier et alii, 2013]. La réussite aux exercices - souvent appelés «tests » par les entreprises - est la garantie que le demandeur d'emploi a les habiletés pour occuper le poste ; elle joue ce faisant le rôle de certification.

«On avait de très bonnes relations avec les missions locales, des fois on faisait passer des tests MRS sur des métiers en tension... par exemple employé libre-service ou autre alors qu'il n'y avait pas forcément des recrutements en cours. Cela permettait d'évaluer ces personnes, de voir qu'elles avaient les habiletés et ainsi, les conseillers en agences étaient sûrs des capacités des personnes et pouvaient les recommander aux employeurs avec un peu plus d'assurance. » (Agent de Pôle emploi)

Les écoles professionnelles sont aussi un canal qui ouvre les frontières du marché. L'alimentation en main-d'œuvre du segment professionnel des métiers de bouches (cuisiniers,

\footnotetext{
${ }^{7}$ « Voilà, c'est un CV, alors « gestionnaire polyvalent », j’ai enlevé, parce que monsieur X va me dire « je ne cherche pas un gestionnaire »... [...] je vais simplement mettre ici « chargé d'accueil ». [...] Oui, la petite A elle est mignonne comme tout, enfin, elle a quand même 37 ans, mais il faut aussi réfléchir plus loin, elle candidate pour des postes commerciaux, dans une banque, elle va me marquer « gestionnaire polyvalent », je ne cherche pas un gestionnaire polyvalent, enfin, la banque ne cherche pas ça, et moi des fois je pense comme la banque.» (Conseillère Pôle emploi, Paris)
} 
pâtissiers, sommelier etc.) jugée difficile dans l'hôtellerie restauration amène à la création de liens étroits entre employeurs et CFA, écoles ou lycées professionnels. En embauchant et en formant des jeunes apprentis ou stagiaires, les établissements indépendants ainsi que les chaînes de prestige, participent à la constitution d'un «vivier» de professionnels [Forté, Monchatre, 2013]. Sur ce segment particulièrement en tension, les employeurs n'ont pas la latitude d'activer un canal qui les relierait à un marché ou à un stock de main-d'œuvre qualifiée qui s'auto-entretiendrait. Recruter nécessite donc de mobiliser un partenaire pour former l'offre de travail dans laquelle il sera ensuite possible de puiser. C'est pourquoi les écoles sont à l'ouest du schéma ${ }^{8}$. Les évaluations scolaires qui ponctuent les formations et la nécessaire formalisation des mises en relation auxquelles participent les établissements expliquent le positionnement sur le versant nord.

Sur ce cadran, les pré-requis et les attentes envers les candidats sont mis en forme, construits avec le terrain professionnel (les entreprises et les formateurs) et non « dictés » par celui-ci. Les pratiques de recrutement des employeurs sont moins figées que sur le cadran nord-est : ils s'adressent aux intermédiaires pour établir des partenariats avec eux, intervenir dans la formation des profils dont ils ont besoin, sortir de leurs routines pour s'ouvrir à de nouveaux profils. L'élaboration d'un langage commun et les coopérations peuvent cependant être longs et compliqués; il y a un risque que recruteurs et intermédiaires ne se comprennent pas et ne parviennent pas à travailler ensemble. Regrettant le caractère chronophage de la méthode, non adapté à leurs besoins urgents de recrutement, plusieurs des entreprises rencontrées avaient renoncé à utiliser la MRS «La MRS? Ça, c'est fini, on ne le fera plus ; c'est une perte de temps, c'est une grosse perte de temps. » (Responsable recrutement, chaîne de restaurants).

\subsection{Compter sur un marché de proximité}

Les canaux situés dans le cadran sud-ouest captent une offre de main-d'œuvre aux contours non définis, soit parce que les entreprises cherchent à s'ouvrir à des profils inhabituels, à élargir leur sourcing et dépasser leurs routines (banque de détail), soit parce que leurs marchés n'ont jamais été organisés au sens de Marsden [1991] (grande distribution et

\footnotetext{
${ }^{8}$ Les écoles sont cependant positionnées moins à l'ouest que la MRS, car il s'agit d'alimenter un marché dont les frontières sont définies par des diplômes et des titres existants.
} 
hôtellerie restauration). Ici cependant, il n'est pas fait appel à des intermédiaires institutionnels pour formaliser les liens et les évaluations, comme dans le cadran du haut. L'information est personnalisée, elle passe par le récit, lors de rencontres en face à face et l'intermédiaire, lorsqu'il y en a, est un salarié ou un proche qui présente personnellement un candidat. Deux grands types de canaux sont situés sur ce cadran : les démarches directes (le candidat se présente en personne dans l'entreprise ou lors d'une opération portes ouvertes) d'une part, et les réseaux personnels, de l'autre. La sélection des candidats sur ce marché de proximité repose alors sur la confiance accordée à l'intermédiaire personnel ou sur les impressions laissées par la première interaction.

Dans la grande distribution, les horaires très matinaux des salariés chargés de la mise en rayon, les coupures éventuelles de la journée de travail en caisse et l'implantation à la périphérie des villes des centres commerciaux, se combinent pour que se dessine une nette préférence pour recruter des personnes habitant à proximité ou/et n'ayant pas de problèmes de locomotion. A cela s'ajoute un important turnover sur ces postes d'employés qui rend nécessaire de recruter en permanence et dans l'urgence. Les candidatures déposées en magasins, de façon spontanée ou en réponse à des annonces placardées en devanture, sont un moyen de capter le «tout-venant » de la main-d'œuvre locale et d'ouvrir sur une pluralité de profils. Plus rare, la publication d'une annonce dans un journal local est une autre réponse à la contrainte de proximité. ${ }^{9}$ Ces candidatures ont de nombreux avantages: elles sont peu coûteuses, arrivent en continu et apportent des candidats «ajustés » aux impératifs de proximité et a priori motivés «Les gens font des démarches de recherche eux-mêmes et on trouve à 95\% notre bonheur dans ces CV.» (Directeur de magasin). Ce canal épouse principalement le format personnalisé du récit. En effet, le CV n'est pas un support essentiel de la mise en relation car en le déposant, le postulant a la possibilité d'échanger verbalement avec le directeur, un adjoint ou même un employé en poste. Cet échange, même très court, permet au recruteur d'avoir des informations essentielles à ses yeux (la disponibilité du candidat, son éventuelle expérience) et d'évaluer en un coup d'œil l'allure et l'attitude du candidat, considérés comme des indices de son aptitude commerciale et son sens relationnel [Nickson et alii., 2012]. Les paroles de cet employé lambda, qui remet à la personne en

\footnotetext{
${ }^{9}$ Ces canaux sont également très utilisés par les établissements des chaînes de la restauration qui rencontrent les mêmes contraintes de proximité, disponibilité et turnover [Forté, Monchatre, 2013].
} 
charge du recrutement d'un hypermarché un $\mathrm{CV}$, sont éclairantes : «Tiens, c'est une petite étudiante qui a déposé son CV ce matin, elle est disponible le lundi et le mardi ». En quelques mots, tout est dit : l'adjectif «petite » témoigne d'un regard bienveillant sur la candidate, on connaît sa disponibilité et on se doute qu'elle sera flexible, les étudiants étant très appréciés pour cela dans ce secteur [Rieucau, Salognon, 2013].

L'exemple de l'opération «portes ouvertes » mise en œuvre par une banque de détail est également instructif, d'autant que ce canal est inhabituel dans ce secteur puisqu'il n'épouse pas le format standardisé du CV mais celui, personnalisé, de l'interaction de visu. La banque a cherché à diversifier le sourcing en organisant des journées portes ouvertes dans des agences parisiennes, avec un entretien immédiat et sans CV comme premier moyen de sélection. Etant donné la compétence recherchée - être commercial -, une évaluation en présence du candidat, plutôt qu'à distance via son CV, paraît pertinente au recruteur. «Dans le cadre d'un échange d'une dizaine de minutes, et s'ils sont des commerciaux, a priori ils vont réussir à nous convaincre d'aller un peu plus loin avec eux»(RH, BDD), ce qui peut amener à retenir un diplômé en STAPS (Sciences et techniques des activités physiques et sportives) qui pourra être formé par la banque, selon sa logique de marché interne. Cette expérience n'a toutefois pas été renouvelée, révélant ici les limites d'un sourcing qui n'est pas médiatisé par des outils de standardisation des candidatures, tels des pages recrutement Internet ou Pôle-emploi. Les recruteurs de la banque, contrairement aux directeurs de magasins, semblent démunis s'ils doivent évaluer de manière décentralisée des personnes qui se présentent sans présélection préalable.

La nécessité d'élargir la «réserve » de main-d'œuvre et de varier les viviers potentiels dans un secteur particulièrement en tension, combinée au peu de ressources consacrées au recrutement, expliquent l'importance que les chaînes de restauration de type économique accordent aux réseaux de proximité, typiques des «liens forts » composés de la famille, des amis ou des proches [Granovetter, 1974]. Les entretiens montrent toutefois que ce canal ne peut fonctionner que si la confiance existe envers la personne qui recommande. A la différence des réseaux professionnels (cadran sud-est), les réseaux de proximité ne portent guère de garantie sur la qualité des candidatures. Ils sont remis en question si un doute s'insère quant à la relation future de travail, par exemple si l'employeur craint que le recrutement de membres de la famille soit porteur de conflits ou que tel salarié ne ramène sa « bande de potes » issu d'un quartier à la mauvaise réputation [Forté, Monchatre, 2013]. 
La méfiance envers les relations de proximité et la non reconduction de l'opération «portes ouvertes » illustrent des difficultés de mise en relation. Dans les deux cas, on peut se demander si le poids du langage ordinaire et l'absence d'intermédiaire spécialisé dans la mise au point ou la traduction des qualités proposées et attendues par les deux parties, ne sont pas à l'origine du manque de confiance et de garanties, impliquant ces semi-échecs.

\subsection{S'adresser à un marché professionnel familier}

Dans le cadran sud-est, l'entreprise s'adresse de nouveau à un marché dont les frontières sont bien stabilisées. Elle sait quelle main-d'œuvre elle recherche mais pour l'atteindre, elle ne passe pas par les canaux du premier cadran qui rendent largement publique sa recherche, mais sollicite discrètement ses réseaux. La question de la confiance dans la personne qui recommande ne se pose pas comme dans le cadran précédent : ici la sélection des canaux s'appuie sur une information personnalisée mais le langage professionnel est partagé et bien compris des partenaires du recrutement. Celui qui recommande et qui joue le rôle d'intermédiaire apporte sa caution, en tant que professionnel, à la candidature : ce sont les réseaux professionnels (HCR, BFI), les cooptations et le recours aux anciens élèves (SSII). Nous sommes sur un marché professionnel [Marsden, 1991] où la main-d'œuvre qualifiée est transférable d'une entreprise à l'autre. Les recruteurs puisent dans un marché déjà constitué et surtout familier: on se connaît dans le «petit monde » de la banque, au sein des réseaux régionaux de l'hôtellerie-restauration, on se reconnaît entre anciens de la même école ou entre collègues informaticiens.

Le secteur HCR est caractérisé par une forte mobilité des salariés d'un restaurant ou d'une entreprise à l'autre et ces salariés en transition représentent une offre dans laquelle puisent les établissements. Les réseaux professionnels sont alors une façon originale d'atteindre cette offre. Les indépendants ont fréquemment recours à ce canal, demandant un avis aux collègues sur telle candidature, dans le cadre de rencontres régulières, de sorte que ce canal est relativement organisé et prend appui sur des codes partagés (le diplôme, l'école où il a été obtenu, l'établissement où la personne a travaillé, etc.). L'information est transmise par le récit, ce qui permet d'expliquer le parcours autrement que ne pourrait le faire un CV déposé sur un site ou dans une agence.

Pour les cadres de la banque de financement et d'investissement (BFI), les relations 
professionnelles (le «petit monde » de la profession), permettent de faire la différence entre des profils qui, sur la base de leur CV, sembleraient identiques. Même lorsqu'un chasseur de tête est rémunéré, ce n'est pas pour proposer un profil original, mais pour aller chercher de manière discrète un profil défini, qui occupe déjà un poste équivalent chez un concurrent ayant les mêmes manières de travailler [Gautié et alii, 2005 ; Larquier, Tuchszirer, 2013]. Un cabinet est a priori un canal formel qui outille la recherche, mais ce qu'attend la banque cliente, c'est sa capacité à mobiliser des réseaux professionnels, d'où son positionnement dans la partie inférieure de notre schéma. Pour les SSII, le canal de la cooptation, contre versement d'une prime, utilise le principe des relations professionnelles repéré dans le petit monde de la BFI, où l'on s'évalue entre pairs, en l'appliquant toutefois dans un milieu de travail moins prestigieux. L'informaticien qui en recommande un autre partage avec celui-ci un langage technique et peut également, avec un langage plus ordinaire, le convaincre de travailler dans sa société plutôt qu'une autre, toutes les «petites » SSII se ressemblant vues de l'extérieur [Fondeur, 2013b] : «Ils nous amènent des gens de profils qui correspondent bien, aussi en termes de culture, parce que ce sont des gens qui se connaissent en général, il y a eu des discussions avant, ces gens-là arrivent avec une bonne connaissance de la société» (Directrice recrutement, SSII)

La sélection opérée par ces canaux est potentiellement forte car sont exclus tous ceux qui n'appartiennent pas aux réseaux. Adopter le bon langage suppose aussi d'être inséré ou présenté par l'un des membres, et non pas seulement de maîtriser les codes ou la langue du métier, comme dans le cadran nord-est. Il y a toutefois plus de possibilités de varier les profils que dans le cadran supérieur car la sélection n'est pas « figée » dans des formats standards.

\section{Conclusion}

Adoptant 1'hypothèse d'offres et de demandes de travail hétérogènes, les analyses économiques appliquées usuelles reconnaissent la diversité des canaux en montrant qu'ils sont spécialisés sur certains profils et en excluent d'autres [Gorter et alii, 1996 ; DeVaro, 2005 ; Sabatier, 2010]. Cette diversité tient pour partie aux ressources diverses dont disposent les acteurs du marché : par exemple, les petites entreprises recourent aux relations personnelles qui ne coûtent rien (et recruteront peu de femmes dépourvues de réseaux professionnels) tandis que les grandes entreprises ont les ressources financières pour rémunérer des cabinets privés (et n'embaucheront pas de chômeur de la sorte). Il n'en demeure pas moins que 
l'homogénéité du fonctionnement du marché du travail et celle des canaux ne sont pas remises en question. Ces derniers peuvent être comparés à l'aune du même étalon, en particulier la vitesse à laquelle ils mènent à un appariement, d'où leur efficacité en termes de durée du chômage ou de la vacance de l'emploi [Sabatier 2003 ; DeVaro, 2005]. Finalement, les canaux font tous la même chose sur le marché du travail (informer sur les opportunités d'emploi, pourvoir des candidatures) avec plus ou moins d'efficacité, en se spécialisant sur des profils «démographiques» au sens large (jeunes/seniors, hommes/femmes, petites/grandes entreprises, etc.).

Ici, dans la lignée des travaux conventionnalistes sur le recrutement et l'intermédiation [Eymard-Duvernay, Marchal, 1997 ; Bessy, Eymard-Duvernay, 1997], nous avons insisté sur la diversité de fonctionnement des canaux en mettant en avant le fait qu'ils s'appuient sur deux investissements de forme pour réduire les incertitudes sur la qualité des appariements. Ils donnent, voire dictent, le format que prend l'information sur les marchés et ils contribuent à façonner les frontières de ces marchés, lorsque celles-ci ne sont pas déjà clairement établies. Pour décrire la pluralité de fonctionnement des canaux, d'autres critères de distinction étaient possibles, en particulier ceux qui caractérisent directement les acteurs de l'intermédiation : leurs organisations internes, leurs groupes professionnels, leurs modèles économiques [Fondeur, Tuchszirer, 2005 ; Fretel, 2012] ${ }^{10}$. Le choix des axes développés ici «constituer une offre versus puiser dans une offre » et «format personnalisé versus format standard» s'explique principalement par le choix méthodologique adoptée par les quatre monographies exploitées. Ces dernières étudient les pratiques de recrutement en privilégiant le point de vue des entreprises et de leurs recruteurs, pour lesquels choisir un canal, c'est choisir quel type d'informations recevoir sur quel type et quel nombre de candidats.

Du point de vue de l'entreprise, le marché du travail n'est pas le lieu où se rencontrent des offres et des demandes (perspective adoptée par le modélisateur en surplomb) mais «le marché externe » auquel elle s'adresse plus ou moins fréquemment, avec plus ou moins d'assurance sur la chance de trouver des candidats correspondant à ses attentes (perspective de l'acteur plongé dans un univers incertain). Les économistes du travail institutionnalistes

\footnotetext{
${ }^{10}$ Remarquons que ces travaux privilégient les intermédiaires institutionnels du marché du travail, au détriment des candidatures spontanées et des réseaux, qui ont pleinement leur place dans notre typologie.
} 
ont peu analysé le marché externe, décrivant plutôt les différents types de marchés internes et laissant penser que la loi de l'offre et de la demande reprenait le pouvoir hors des murs de l'entreprise. De notre côté, nous partons du constat que l'entreprise qui rentre sur le marché du travail pour rencontrer son offre de travail, le fait très concrètement en activant un canal. Par conséquent, le marché externe est pourvu des caractéristiques que lui donne le canal activé : ses frontières coïncident avec celles de l'offre de main-d'œuvre qu'atteint le canal et il est décrit par l'information mise en forme par le canal. C'est ainsi que caractériser des canaux et leurs manières de sélectionner la main-d'œuvre revient à décrire des marchés du travail aux fonctionnements distincts. Une lecture radicale dirait que les marchés externes n'existent pas ; il n'existe que des canaux qui, sélectionnant la main-d'œuvre, façonnent ce qui est appelé l'« offre de travail ».

De manière moins radicale, dans ce travail, nous avons qualifié le recours à tel ou tel canal comme une manière spécifique pour une entreprise de s'adresser à son marché externe. L'entreprise peut ainsi : envoyer et recevoir des informations sur un marché du travail standard; demander à un intermédiaire institutionnel de façonner une nouvelle maind'œuvre; compter sur un marché de proximité; ou encore, s'adresser à un marché professionnel familier. A chaque fois, l'offre de travail est façonnée différemment. Les canaux du marché standard sélectionnent sur des critères stabilisés, voire durcis, tels le diplôme et l'expérience, qui peuvent être excluant. Lorsqu'il y a recours et partenariat avec un intermédiaire institutionnel, les pré-requis et les attentes envers les candidats sont mis en forme avec le terrain professionnel (les entreprises et les formateurs) et non « dictés »par celui-ci. Lorsque l'entreprise compte sur un marché de proximité, la sélection repose sur la confiance accordée à celui qui recommande. Cette fois, c'est l'absence de réseaux de proximité «de qualité » qui exclut. Enfin, quand l'entreprise s'adresse à un marché professionnel familier, la question de la confiance dans la personne qui recommande ne se pose pas comme dans le cas précédent: ici la sélection des canaux s'appuie sur une information personnalisée mais le langage professionnel est partagé et bien compris des partenaires du recrutement.

Contrairement à la littérature économique standard qui cherche à classer les canaux selon leur efficacité (ce qui, répétons-le, suppose qu'ils font la même chose sur le marché du travail), aucune des manières de s'adresser au marché n'est présentée ici comme «le » 
modèle à suivre, celui qui serait le plus efficace pour optimiser les rencontres entre employeurs et candidats, puisque canaux, marchés, entreprises et candidats ne peuvent être compris hors des interactions qu'ils entretiennent. Il est difficile par exemple de concevoir un jobboard - typique du cadran nord-est - sur un marché de proximité non organisé (cadran sud-ouest) : la mise en forme très standardisée de l'outil Internet risquerait d'être soumise à la critique des acteurs, soulignant les limites du langage informatique face à la richesse de la recommandation portée par le discours d'une personne ou de la candidature déposée spontanément par un chercheur d'emploi motivé et résidant à proximité [Rieucau, 2013]. Cela ne signifie pas que rien ne puisse bouger mais que l'entreprise qui change de canal, change de marché, de main-d'œuvre ciblée et modifie l'ensemble de son processus de recrutement.

\section{Références bibliographiques}

AUTOR D.H. (2009), The Studies of Labor Market Intermediation, University of Chicago Press, $456 \mathrm{p}$.

BenarRosh Y. (2000), «Tri des chômeurs: le nécessaire consensus des acteurs de 1'emploi», Travail et emploi, n81, p. 9-26

Bessy C., EyMARD-Duvernay F. (éds) (1997) Les intermédiaires du marché du travail, Paris, PUF

Bessy C., LARQuier G. DE (2010), «Spécialisation et efficacité des intermédiaires du placement », Revue française d'économie, 2(25), p. 226-270

Bessy C., Marchal E. (2009), «Le rôle des réseaux et du marché dans les recrutements. Enquête auprès des entreprises », Revue française de socio-économie, n ${ }^{3}$, p. 121-146

Bouchardeau C., CApelier T. (2010), «Evaluation de la méthode de recrutement par simulation », Repères et Analyses, $\mathrm{n}^{\circ} 16$, Pôle emploi

BuREAU M.C, MARChAl E. (2009), «Incertitudes et médiations au cœur du marché du travail », Revue Française de Sociologie, 50(3), p. 573-598

Cahuc P., Fontaine F. (2004), «Le rôle des allocations chômage en présence de différentes méthodes de recherche d'emploi », Revue économique, vol.55 n³, p.591-600

Chauvin S. (2010), Les agences de la précarité, Seuil, Paris

DeVAro J. (2005) «Employer Recruitment Strategies and the Labor Market Outcomes of New Hires », Economic Inquiry, vol. 43, p. 263-282 
DIVAY S. (2009), « Nouveaux opérateurs privés du SPE : les pratiques des conseillers sontelles novatrices? », Travail et Emploi, ${ }^{\circ}$ 119, p. 37-49

DOERINGER P.B, PIORE J.M (1971), Internal Labour Market and Manpower Analysis, D.C Heath and company, Lexington, Massachusetts

Duguet E., LÉAndri N, L’Horty Y., Petit P. (2007), «Le testing, une méthode expérimentale de mesure des discriminations à l'embauche », Note de Veille, ${ }^{\circ} 48$, Centre d'Analyse Stratégique

Dunlop J. (1966), «Job vacancy Measures and Economic Analysis», The Measurements and Interpretation of Job Vacancies, National Bureau of Economic Research, Columbia University Press, p. 27-48

Eymard-Duvernay F., MARChal E. (1997), Façons de recruter. Le jugement des compétences sur le marché du travail, Métaillé, Paris

FONDEUR Y. (2013a), «Introduction- Systèmes d'emploi et pratiques de recrutement », La revue de l'Ires $\mathrm{n}^{\circ} 76$, p. 31-43

FONDEUR Y. (2013b), «Services de conseil en informatique : recruter pour placer », La revue de l'Ires $\mathrm{n}^{\circ} 76$, p. 99-125

FondeUR Y., LARQUIER G. DE (Eds) (2012), «Pratiques de recrutement et sélectivité sur le marché du travail », Rapport de recherche du CEE $\mathrm{n}^{\circ} 72$

FONDEUR Y., TUCHSZIRER C (2005) Internet et les intermédiaires du marché du travail, Rapport IRES pour l'ANPE

Forté M., Monchatre S. (2013), «Recruter dans l'hôtellerie-restauration : quelle sélectivité sur un marché du travail en tension ? », La revue de l'Ires n76, p. 127-150

FrETEL A. (2012), «Typologie des figures de l'intermédiation Quelle relation à l'entreprise au delà de l'impératif adressé aux opérateurs du SPE ? », Document de travail IRES n ${ }^{\circ} 2$

FRETEL A. (2013), «La notion d'accompagnement dans les dispositifs de la politique de 1'emploi : entre centralité et indétermination », Revue Française de Socio-Economie, n ${ }^{\circ 1}$, p. $55-79$

Gautié J., Godechot O., Sorignet P.E. (2005), «Arrangement institutionnel et fonctionnement du marché du travail : le cas de la chasse de tête », Sociologie du travail, 47, p. $383-404$ 
Gorter C., NijkamP P., Rietveld P. (1996) «Employers' Recruitment Behaviour and Vacancy Duration: an Empirical Analysis for the Dutch Labour Market», Applied Economics, vol. 28, n¹1, pp. 1463-74

GrANOVETTER M.S. (1974), Getting a job. A study of contacts and careers, The University of Chicago Press, USA. Première edition, 1974, edition augmentée, 1995

IOANNIDES Y., DATCHER LOURY L. (2004), «Job Information Networks, Neighbourhood Effects and Inequalities », Journal of Economic Literature, XLII, p. 1056-1093

LAgARENNE C., MARChal E. (1995), «Recrutements et recherche d'emploi » La lettre du Centre d'études de l'emploi, $\mathrm{n}^{\circ} 38$, juin

LARquier G. DE, Marchal E. (2012), «La légitimité des épreuves de sélection : apports d'une enquête statistique auprès des entreprises », in F. Eymard-Duvernay (éd.), Epreuves d'évaluation et chômage, Octarès éditions, p. 47-77

LARQuiER G. DE, TUCHSZIRER C. (2013), «Le secteur bancaire : des recrutements sous l'autorité des ressources humaines ? », La revue de l'Ires nº76, p. 71-98

LARQuier G. DE, RieuCAu G., TuChSZIRER C. (2013), «La Méthode de recrutement par simulation de Pôle emploi : jusqu'où les entreprises se l'approprient-elles ? », Connaissance de l'emploi, CEE, $\mathrm{n}^{\circ} 107$

LATSIS J., BESSIS F., LARQUIER G. DE (2010), «Are conventions solutions to uncertainty? Contrasting visions of social coordination », Journal of Post Keynesian Economics, vol.32, $\mathrm{n}^{\circ} 4$, p. $335-558$

LOCKYeR C., SchOlarios D. (2004), «Selecting hotel staff: why best practice does not always work », International Journal of Contemporary Hospitality Management, 16(2), p. $125-135$

MARCHAL E., RIEUCAU G. (2010), Le recrutement, la découverte, Repères

Marchal E., Mellet K., Rieucau G. (2007), « Job board toolkits: Internet matchmaking and changes in job advertisements », Human Relations, vol. 60 (7), p. 1091-1113

MARSDEN D. (1991), «Action syndicale et structures des marchés du travail », CFDTAujourd'hui, $\mathrm{n}^{\circ} 102$, septembre, p. 43-56

Nickson D, Warhurst C, Commander J, Hurrell SA., Cullen AM. (2012), « Soft skills and employability: Evidence from UK retail», Economic and Industrial Democracy, 33 (1), p. 65-84 
PARADEISE C. (1988), «Les professions comme marchés du travail fermés », Sociologie et sociétés, 20(2), p. 9-21

PIORE, M., SAFFORD, S. (2007), «Preliminary Thoughts on Identity and Segmentation in Primary sector Labor Markets », Economies et Sociétés, Série Socio-Economie du travail, $\mathrm{AB}, \mathrm{n}^{\circ} 28$, p. $925-940$

REES A. (1966), «Labor economics: effects of more knowledge. Information networks in labor markets », American Economic Review, 56(2), p. 559-566

RIEUCAU, G. (2013), «Etre employé dans la grande distribution : candidater en personne ou en ligne? $\gg$ Connaissance de l'emploi, CEE, $\mathrm{n}^{\circ} 104$

RIEUCAU G., SAlOGNON M. (2013), «Le recrutement dans la grande distribution : des pratiques ajustées ?, La revue de l'Ires $n^{\circ} 76$, p. 45-69

SABATIER M. (2003), «Stratégies d'insertion et durée d'accès au premier emploi: une analyse micro-économique sur le panel téléphonique du CEREQ (1989-93)», Revue d'économie politique, $\mathrm{n}^{\circ} 5$, p. 671-696

SABATIER M. (2010), «Filling vacancies: identifying the most efficient recruitment channel », Economics Bulletin, 30(4), p. 3355-3368

SPENCE M. (1973), «Job Market Signaling », TheQuarterly Journal of Economics, Vol. $87 ; n^{\circ} 3$, p. $355-374$

STIGLER J. (1962), «Information in the Labor Market» The Journal of Political Economy, vol.70 part 2, p. $94-105$

ThÉVEnot L. (1985), «Les investissements de forme» in Conventions économiques CEE/PUF, Paris, p. 21-72

ThÉvenot L. (1997), «Un gouvernement par les normes. Pratiques et politiques des formats d'information » in Conein et Thévenot, (Eds), Cognition et information en société, Paris, Edition de l'EHESS 